\title{
Design and Implementation of a Capacitance Based Air Bubble Detection System for Hemodialysis Machine
}

\author{
Pratondo Busono ${ }^{1}$ \\ ${ }^{1}$ Center for Pharmaceutical and Medical Technology \\ Agency for the Assessment and Application of Technology \\ Jakarta, Indonesia \\ e-mail: prabusono@yahoo.com
}

\begin{abstract}
Hemodialysis is one of the medical treatment methods for the patients with end stage renal disease. It is conducted through the use of artificial kidney, located outside of the human body. During the hemodialysis therapy, small air bubbles may infiltrate the blood tubing and coalesce to perform the larger bubble which can be harmfull for the patient if entering the patient's blood circulatory system.

The objective of this work is to develop an capacitance based air bubble detection system for hemodialysis machine. The research method covers conceptual design, detail design, prototyping and performance testing for the capacitance sensor as well as the data acquisition system.

The validation test was conducted to verify the functionality of the system. For testing purposes, an experimental setup was constructed and several test runs were conducted. Comparison between measurement conducted using capacitance technique and using ultrasonic technique was also presented in this work. It shows that close agreement between the results obtained using ultrasonic technique and capacitance technique is observed in this work.
\end{abstract}

Keywords-bubble detector; capacitance technique; hemodialysis machine.

\section{INTRODUCTION}

Kidneys are the vital organs for the human body. They have importan functions such as removing blood from the body, filtering and treating the blood to remove toxic substance, regulating the blood pressure, reestablishing proper chemical levels such ion and $\mathrm{pH}$ level in the blood. Every minutes, approximately $1200 \mathrm{ml}$ of the blood passes through the kidneys [1].

People diagnosed with end stage renal disease (ESRD) can undergo either hemodialysis or CAPD. However, most of the patients prefer hemodialysis treatment. The hemodialysis treatments are performed through the use of a dialyzer, which is also known as an artificial kidney. The blood is transported from the patient to the dialysis machine through a set of plastic tube and blood flow through the machine is driven by a peristaltic pump. During treatment the patient has got two needles in one arm. One is known as the arterial needle, where the blood flow is directed towards the dialysis machine. The second is called the venous needle and here the cleansed blood is returned to the body. During the transportation, small air bubbles may infiltrate the blood tubing and coalesce to perform the larger bubble which can be harmfull for the patient. If an air bubble is large enough and enters the patient's blood circulatory system, the blood flow can be blocked and the patient can die by air embolism [2].

The objective of this work is to develop a capacitance based air bubble detector hemodialysis machine. The research method used in this work includes conceptual design, detail design, system validation and performance testing for capacitance sensor and the data acquisition system. The hardware system consists of capacitance sensor, analog and digital circuitry and I/O interface.

The validation test was conducted to verify the functionality and validity of the system. For testing purposes, an experimental setup was constructed. It consist of simple flow loop with peristaltic pump, air bubble injector, capacitance sensor modul, blood line clamp, and electronic circuitry. The artificial blood (dextran) was used instead of human blood. Three bubble sizes were used for the experiments: $50 \mu \mathrm{L}, 75 \mu \mathrm{L}$ and $100 \mu \mathrm{L}$. For each experiments, 10 test runs were conducted. The capacitive signals for various bubble size obtained in this work. Comparison between measurement conducted using capacitance method and ultrasonic method was presented in this work.

\section{THEORY}

Bubble detection using capacitance technique is based on on the fact that the difference in permittivity of the different components or phases flowing between two capacitance plates (electrodes) makes the capacitance between these two plates dependent on the ratio of concentration of the components or the phases in the flow. In addition, such a capacitance is also dependent on the flow regimes. Even if the capacitance technique is flow regime dependent it can still be used for the concentration measurements if the components are homogeneously mixed [3].

Although, the basic concept of a capacitance transducer is very simple, but in real application there are many factors that influence the measurements in such a way that the 
results can easily be miss-interpreted if the basic principles are not thoroughly understood. The simplest capacitance sensor consists of two parallel metal plates separated a distance from each other. The first plate is called as exitation electrode and the second plate is called as sensing electrode. The capacitance between the two parallel electrode can be written as [4]

$$
C_{s}=\frac{\varepsilon_{r} \varepsilon_{0} A}{d}
$$

where $A$ is the area of the sensing electrode $\left(\mathrm{m}^{2}\right)$

$d$ is the distance between the electrodes (m)

$\varepsilon_{r}$ is relative permittivity of the material between the electrode plates

$\varepsilon_{o}$ is permittivity of free space $\left(8.854 \cdot 10^{-12} \mathrm{~F} / \mathrm{m}\right)$

The electric field between these two electrode plates usually is not be homogeneous and it will be more sensitive to concentration variations in the surroundings of the gaps between the plates. The guard electrode is used to eleminate the the influence of the strongest inhomogeneous field at the edges of the electrodes. If the guard electrodes are kept at the same potential as the sensing electrode but electrically insulated from it, the influence of the fringe field or edge effect of the electrodes can be eliminated.

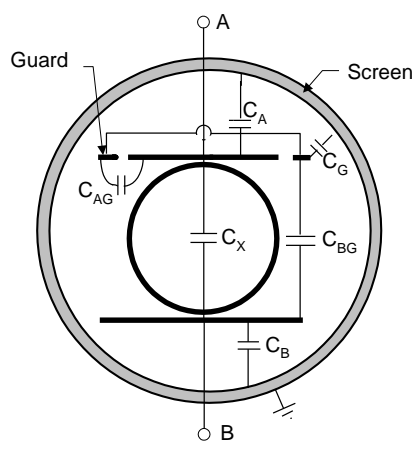

(a)

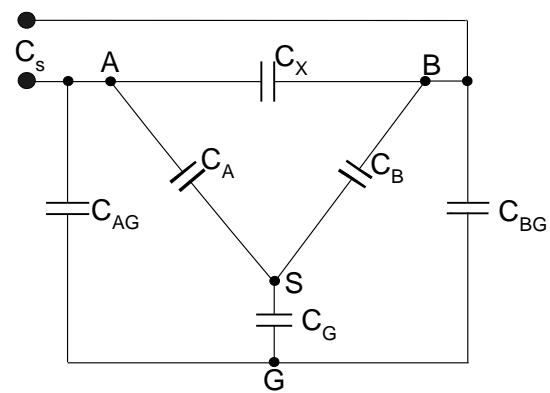

(b)

Figure 1. a) Capacitance sensor with guard electrode and screen. Electrode A is the detector electrode and electrode B is the excitation electrode, b) equivalent digram of capacitance sensor [3].

In order to perform as accurate measurements as possible it is necessary to both guard and screen the capacitance sensor as shown in Figure 1(a) with the equivalent diagram of a capacitance sensor shown in Figure 1 (b). For all practical sizes of sensors the parasite capacitances $C_{A}$ and $C_{B}$ are considerable, thus resulting in an unwanted shunting of $\mathrm{C}_{\mathrm{X}}$. It is also a disadvantage that both $C_{A}$ and $C_{B}$ will change when the permittivity of the mixture in the pipe changes due to displacement of the field lines between the electrodes and the screen. However, the influence of all the parasite capacitances can be diminished if a proper guard-driver detector is used [3].

To eliminate the influence of the various parasite capacitances in the sensor head, the virtual ground circuit method is used. Figure 2 shows the basic principle of this circuit. It can easily be seen that if $A_{O L}$ is large, the

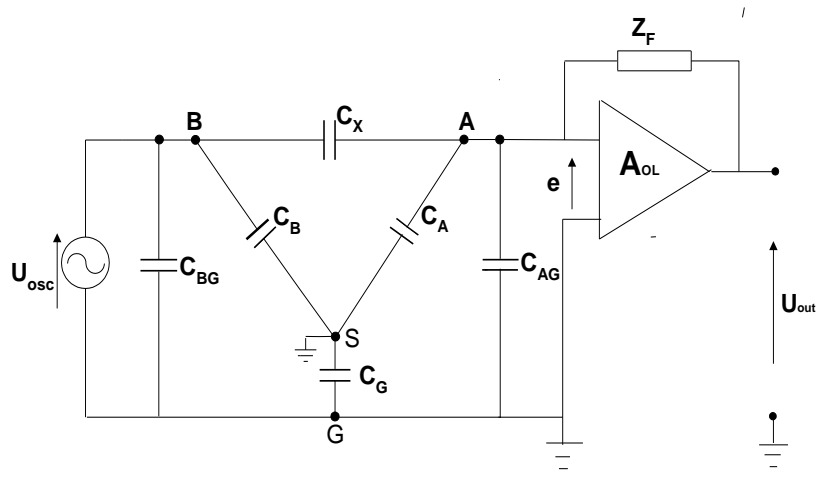

Figure 2. Capacitance transducer with grounded guard and screen and guarded electrode (A) connected to a virtual ground circuit [3].

differential input voltage $e \approx 0$ and the output signal of the circuit is:

$$
u_{\text {out }}=u_{o s c} z_{F} j \omega C_{X}
$$

and if the feedback impedance $\mathrm{Z}_{\mathrm{F}}$ is a capacitor $\mathrm{C}_{0} \quad\left(\mathrm{Z}_{\mathrm{F}}=\right.$ $1 / \mathrm{j} \omega \mathrm{C}_{0}$ ) equation ( 3 ) can be written:

$$
u_{\text {out }}=u_{\text {osc }} \frac{C_{x}}{C_{0}}
$$




\section{MATERIAL AND METHOD}

Methods used in this work include sensor development, electronic development, implementation and validation.

\section{A. Sensor Development}

As mentioned in the previus section, that the capacitance is not only dependent on the flow regime and dielectric constant of the medium, but also the shape of the electrodes. In this work, concave electrode configuration was tested. The reason of chosing this concave configuration is much more suitable for hemodialysis machine application. This shape can be directly integrated to the bubble detector housing installed in most hemodialysis machine. Figures 3 (a) and (b) shows the main dimension of the sensor.

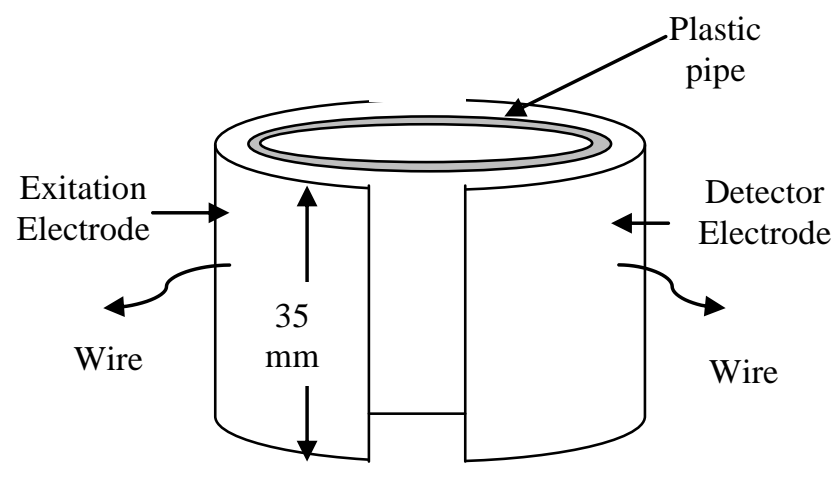

(a)

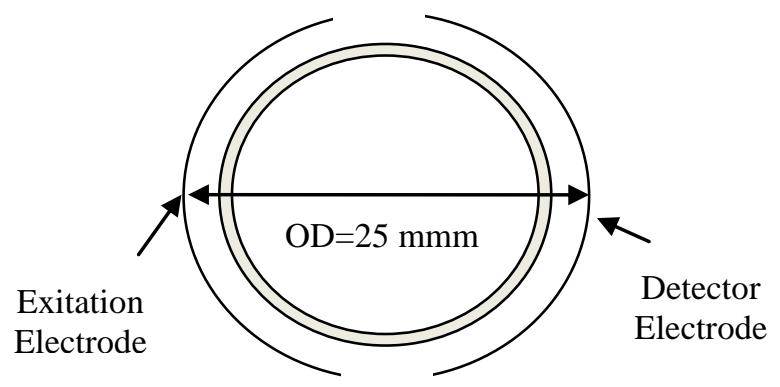

(b)

Figure 3. Concave shape capacitance sensor

\section{B. Electronic Development}

The electronic system has two main functions; for controlling the capacitance sensor as well as for measuring and displaying the capacitance signals. The system consists three main parts: signal generator circuitry, analog and digital signal circuitries (data acquisition system). The block diagram of the system is shown in Figure 4. The signal generator circuitry is used to generate signal which shape, amplitude and measuring frequency can be adjusted. The measuring frequency used in the system is $500 \mathrm{~Hz}$. The sensor had to be sensitive enough to detect tiny fluctuation in capacitance.

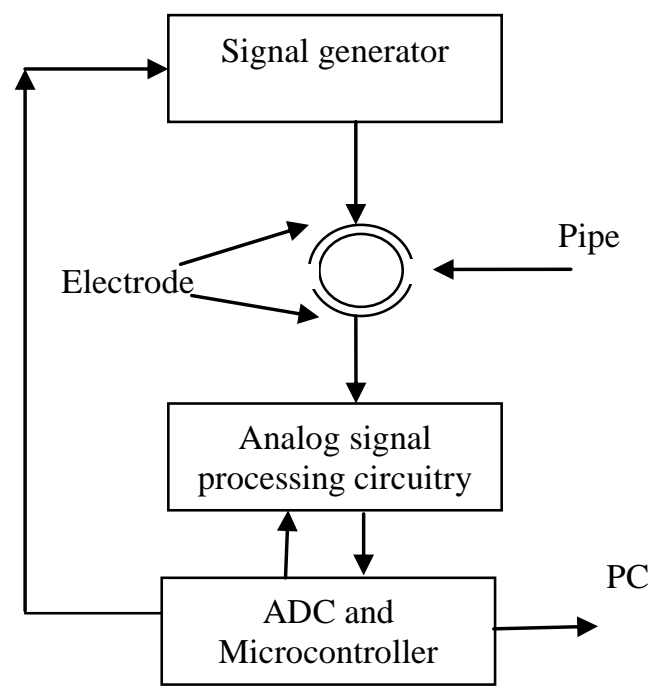

Figure 4. Block diagram of electronic system

The resulting design that was thought to comply with all these requirements was based on an RC-oscillator circuit. Such an oscillator has the benefit of producing a capacitance dependant frequency that can be easily and accurately detected using a data-acquisition card connected to a computer. An RC-oscillator circuit also provides a very simple relationship between the frequency and capacitance, as shown in Eq. 5 [3],

$$
f=\frac{1}{2 \pi R C}
$$

The capacitance waveform is fed into an analog signal processing circuitry, which consists of high speed operational amplifier gain system, filter and voltage offset stages. The amplified signal is then fed into a second order anti-aliasing filter. This filtered signal is then offset to condition the signal for the A/D converter input span voltage. The analog processed signal is converted to a digital waveform by a high speed, analogue-to-digital converter embedded in the ATMega32 Microcontroller.

After digitized, the sampled data is stored in RAM for post processing. The microcontroller then streams the data to the PC for further processing which is to reveal the content of the waveform..

\section{Implementation}

The prototype of electronic system had been developed in this work. It consists of analog and digital circuitry for 
controlling the signal generator as well as for detecting the presence of air bubble. The embedded software has been integrated to the hardware.

\section{Validation}

The validation test was conducted to verify the functionality of the system. For testing purposes, an experimental flow loop was constructed. It consist of simple flow loop with peristaltic pump, air bubble injector, bubble detector modul, tube clamp, and electronic circuitry as shown in Figure 5.

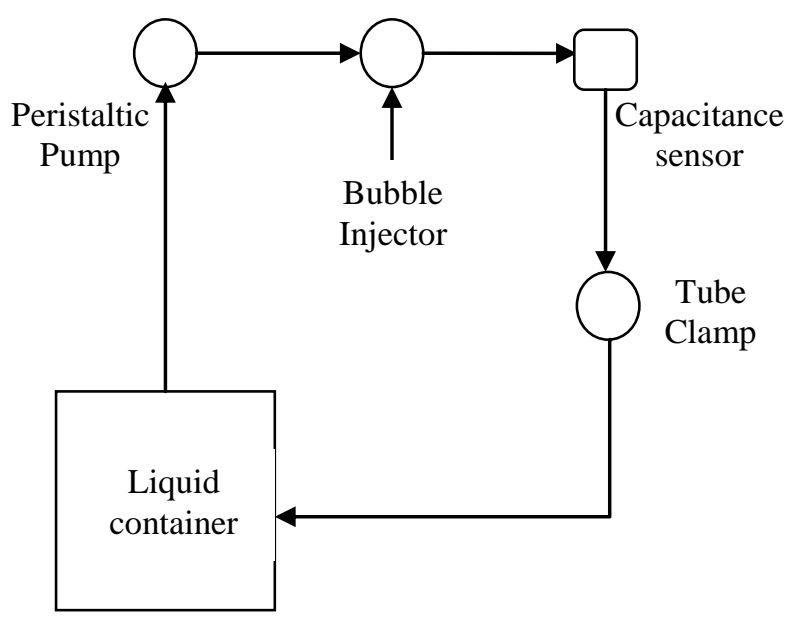

Figure 5. Experimental Setup

The artificial blood (Dextran 70 solution) was used instead of human blood. Three bubble sizes were used for the experiments: $50 \mu \mathrm{L}, 75 \mu \mathrm{L}$ and $100 \mu \mathrm{L}$. For each experiments, 10 test runs were conducted. The capacitance signals was recorded for various bubble sizes. Comparison between measurement using ultrasonic and capacitance was also performed in this work.

\section{RESULTS}

\section{A. Calibration Curve}

The capacitance sensor was based on the AC method as discussed by Mariolli et al. [5], however, without the feedback circuitry. It was developed and assembled for hemodialysis machine application. An HIOKI LCR meter was used for calibrating the sensor with the exitation frequency of $10 \mathrm{kHz}$. The uncertainty of the measurement was $\pm 0.03 \mathrm{pF}$ with confidece interval of $95 \%$. The experiment was conducted at ambient temperatur of $37^{\circ} \mathrm{C}$ Figure 6 shows the calibration curve for the sensor.

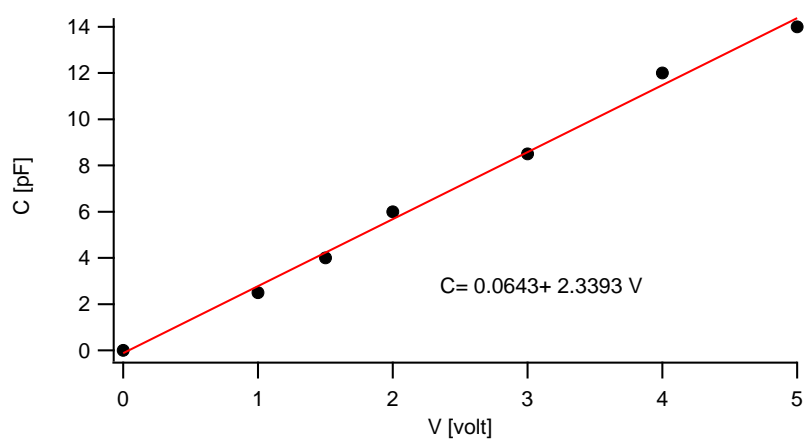

Figure 6. Calibration curve for the capacitance sensor

Figure 7 shows the response of capacitance sensor for various bubble sizes. It shows that the capacitance tends to decrease with the increasing bubble sizes.

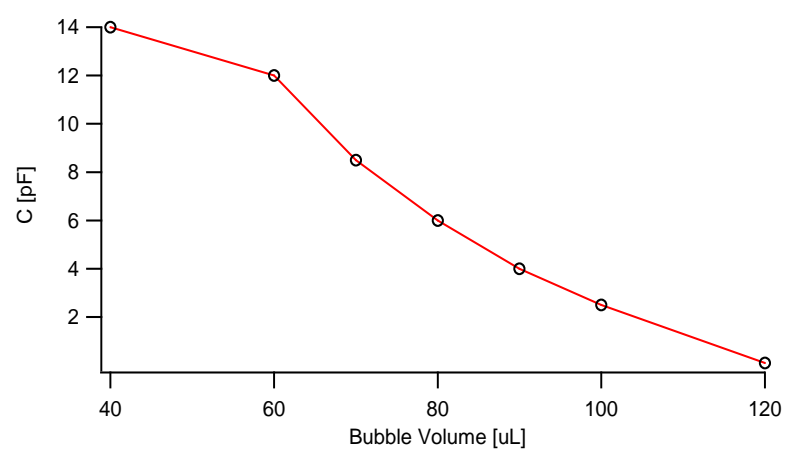

Figure 7. Capacitance as a function of bubble volume

\section{B. Recorded Signals}

Figure 8 shows amplitude of the recorded capacitance signals for bubble size of $75 \mu \mathrm{L}$. The present of the bubbles can seen by the present of peaks. It can be seen from the bottom plot that the higher size of bubble, the higher amplitude of the waves.

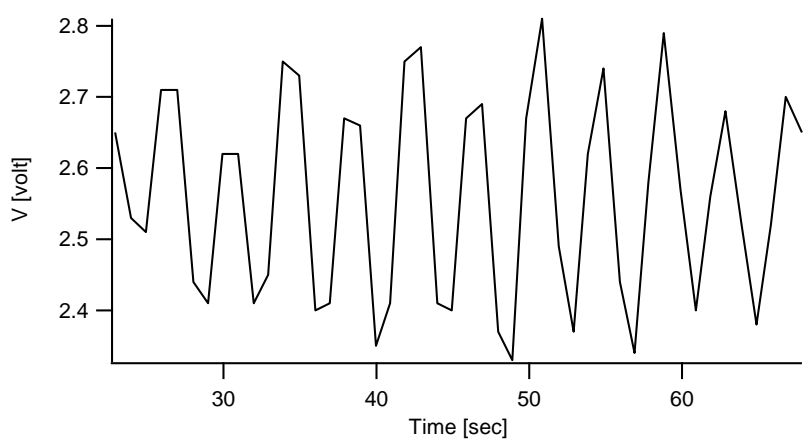


Figure 8. Capacitance signal amplitudes for $75 \mu \mathrm{L}$ bubble size

Table 1 shows the comparison between bubble detection conducted using the capacitance sensor and the ultrasonic sensor. It demonstrates that for small bubble size less than 50 $\mu \mathrm{L}$, capacitance sensor fails to detect compared using ultrasonic sensor.

Table 1. Comparison between results obtained using capacitance and ultrasonic sensor

\begin{tabular}{|c|c|c|c|}
\hline \multirow{2}{*}{ No } & \multirow{2}{*}{$\begin{array}{c}\text { Bubble Volume } \\
(\mu \mathrm{L})\end{array}$} & \multicolumn{2}{|c|}{ Alarm } \\
\cline { 3 - 4 } & 50 & Capacitance & Ultrasonic \\
\hline 1 & 75 & No & Yes \\
\hline 2 & 100 & Yes & Yes \\
\hline 3 & & Yes & Yes \\
\hline
\end{tabular}

\section{CONCLUSION}

The system suggests that bubble detection using capacitance sensor is not only reasonable from a physics point of view, but also form an integrated electronics point of view.

The real-time implementation can be achieved by installing the capacitance sensor into the simple flow loop and it shows that the capacitance sensor seems to be the promising low cost sensor for detecting the air bubble for hemodialysis machine application.

Some improvement should be made to improve the sensitive of the sensor and the electronic system.

\section{ACKNOWLEDGMENT}

The research is partly founded by the Ministry of Research and Technology, Republic of Indonesia, through Research Grant Insentif Riset SINas 2004 No. RT 2014-1265 "Pengembangan Teknologi Mesin Hemodialysis Untuk Mendukung Pelayanan Pasien Gagal Ginjal di Rumah Sakit".

\section{REFERENCES}

[1] M. Misra, The basics of hemodialysis equipment, Hemodialysis International, 9: 30-36 (2005).

[2] D. Woltmann, A.R. Fatica, M.J. Rubin and W. Weitzel, "Ultrasound detection of micro embolic signals in hemodialysis accesses," American Journal of Kidney Diseases, vol. 35, 2000, pp. 526- 528.

[3] E.A. Hammer, "Capacitance transducers - Basic Principles" Multiphase Flow Handbook, 2003. Crowe, C (editor), Multiphase Flow Handbook, (Taylor and Francis Group) ISBN 0-8493-1280-9.

[4]. L. K. Baxter, "Capacitive sensors; design and applications" IEEE, New York, 1997.
[5]. D. Mariolli, E. Sardini, A. Taroni, "High-accuracy measurement techniques for capacitance transducers". Measurement Science and Technology, v. 4 ,n. 3, 1993, p. 337-343. 